\title{
GH modulates hepatic epidermal growth factor signaling in the mouse
}

\author{
Lorena González, Ma Eugenia Díaz, Johanna G Miquet, Ana I Sotelo, Diego Fernández , \\ Fernando P Dominici, Andrzej Bartke ${ }^{2}$ and Daniel Turyn
}

Departamento de Química Biológica, Facultad de Farmacia y Bioquímica, Instituto de Química y Fisicoquímica Biológicas (UBA-CONICET),

Universidad de Buenos Aires, Junín 956, 1113 Buenos Aires, Argentina

${ }^{1}$ Cátedra de Bioquímica Humana, Facultad de Medicina (UBA), Buenos Aires, Argentina

${ }^{2}$ Geriatrics Research, Departments of Internal Medicine and Physiology, School of Medicine, Southern Illinois University, Springfield, Illinois 62702, USA

(Correspondence should be addressed to L González; Email: Igonza74@yahoo.com.ar)

\begin{abstract}
Epidermal growth factor (EGF) is a key regulator of cell survival and proliferation involved in the pathogenesis and progression of different types of cancer. The EGF receptor (EGFR) is activated by binding of the specific ligand but also by transactivation triggered by different growth factors including GH. Chronically, elevated GH levels have been associated with the progression of hepatocellular carcinoma. Considering EGF and GH involvement in cell proliferation and their signaling crosstalk, the objective of the present study was to analyze GH modulatory effects on EGF signaling in liver. For this purpose, $\mathrm{GH}$ receptor-knockout (GHR-KO) and GH-overexpressing transgenic mice were used. EGFR content was significantly decreased in GHR-KO mice. Consequently, EGF-induced phosphorylation of EGFR, AKT, ERK1/2, STAT3, and STAT5 was significantly decreased in these mice. In contrast, EGFR content as well as its basal tyrosine phosphorylation was
\end{abstract}

increased in transgenic mice overexpressing GH. However, EGF stimulation caused similar levels of EGFR, AKT, and ERK1/2 phosphorylation in normal and transgenic mice, while EGF induction of STAT3 and STAT5 phosphorylation was inhibited in the transgenic mice. Desensitization of the STATs was related to decreased association of these proteins to the EGFR and increased association between STAT5 and the tyrosine phosphatase $\mathrm{SH}$ 2-containing phosphatase-2. While GHR knockout is associated with diminished expression of the EGFR and a concomitant decrease in EGF signaling, GH overexpression results in EGFR overexpression with different effects depending on the signaling pathway analyzed: AKT and ERK1/2 pathways are induced by EGF, while STAT3 and STAT5 activation is heterologously desensitized.

Journal of Endocrinology (2010) 204, 299-309

\section{Introduction}

The relevance of growth factors to the pathogenesis of human cancer has long been established. Different mechanisms may contribute to amplify the signal driven by growth factors: the overexpression of growth factors or the overexpression and/or hyperactivation of their receptors or molecules involved in their signaling cascades. Among the growth factors and growth factor receptors that have been shown to be involved in the pathogenesis and progression of different carcinoma types is the epidermal growth factor (EGF) family of peptide growth factors and the EGF receptor (EGFR; Ito et al. 2001, Normanno et al. 2001, 2006).

EGFR (ErbB-1) belongs to a family of receptors, which comprises three additional proteins ErbB-2, ErbB-3, and ErbB-4. ErbB receptors are activated by binding of growth factors of the EGF family, which are produced in an autocrine or paracrine way. EGF binding to its receptor leads to ErbB1 homodimerization or heterodimerization with the other ErbB receptors (Jorissen et al. 2003). This leads to the activation of the receptor tyrosine kinase domain and phosphorylation of multiple tyrosine residues within their intracellular domains (Boeri Erba et al. 2005, Wu et al. 2006). EGFR phosphorylation leads to recruitment of a number of docking and signaling proteins such as Grb-2, SHC, protein tyrosine phosphatase (PTP)-1B, PLC $\gamma$ and SRC, among others. These interactions trigger intracellular signaling cascades such as the Ras/RAF/MEK/ERK (p44/p42 MAPK), p38 MAPK, the PKC, the PI3K/AKT, and the STAT pathways, involved in cell proliferation, survival, and motility (Jorissen et al. 2003, Henson \& Gibson 2006, Normanno et al. 2006).

ErbB receptors are activated not only by direct binding of specific ligands but also by transactivation. Growth factors such as GH and prolactin (PRL) can indirectly activate ErbB receptors through tyrosine kinase JAK2 (Yamauchi et al. 1997, 1998, 2000). GH was first described to induce Tyr1068 EGFR phosphorylation both in vitro and in vivo (Yamauchi et al. 1997, 1998). GH was also shown to induce EGFR phosphorylation at residues Tyr845, Tyr992, and Tyr1173 in mouse preadipocytes (Kim et al. 1999, 
Huang et al. 2003). GH and PRL can also control EGFR turnover by threonine phosphorylation, thus modulating EGFR signaling (Kim et al. 1999, Huang et al. 2003, 2004).

$\mathrm{GH}$ is a pituitary hormone involved in longitudinal growth promotion and metabolic processes. $\mathrm{GH}$ binding to its receptor (GHR) triggers enhanced association with and activation of JAK2, which subsequently phosphorylates diverse signaling mediators (Waters et al. 2006, Lanning \& Carter-Su 2007, Brooks et al. 2008). Three major signaling systems are activated in response to GH: the STATs, PI3K/ AKT, and p44/p42 MAPK (ERK1/2) signaling pathways. Concerning the STATs, GH has been reported to activate STAT1, STAT3, and, mainly, STAT5, which regulates the transcription of the insulin-like growth factor 1 (Igf1) gene (Zhu et al. 2001, Woelfle \& Rotwein 2004).

Several recent studies have suggested that, in addition to its effects on growth and metabolism, GH is involved in tumorigenesis and tumor progression. GH overexpression has been associated with cancer in animal models as well as in humans; indeed, acromegalic patients show an increased incidence of this pathology (Webb et al. 2002, Jenkins 2004, Siegel \& Tomer 2005). Moreover, proliferative diseases in humans have been associated with the overexpression of $\mathrm{GH}$ (Raccurt et al. 2002). Transgenic mice overexpressing GH are more susceptible to develop cancer (Orian et al. 1990, Wanke et al. 1991, Snibson 2002) and they have an increased tendency to develop hepatocellular carcinoma at advanced ages (Snibson 2002, Bartke 2003). On the contrary, absence or low GH levels are associated with reduced tendency to develop malignancies spontaneously (Anisimov 2001, Ikeno et al. 2003) or in response to administration of carcinogens (Styles et al. 1990, Pollak et al. 2001).

The increased susceptibility of transgenic mice overexpressing $\mathrm{GH}$ to develop liver cancer could be attributed to high levels of circulating IGF1. However, IGF1 has been described to induce discrete metabolic effects and only a slight increase of DNA synthesis in liver (Hartmann et al. 1990, Kimura \& Ogihara 1998, Grunnet et al. 1999). Moreover, IGF1 binding to hepatocytes is barely detectable (Barreca et al. 1992, Santos et al. 1994). What is more, transgenic mice overexpressing IGF1 do not show the hepatic histopathological alterations that are observed in the liver of GH-overexpressing transgenic mice (Bartke 2003). Even when up-regulation of proliferative and anti-apoptotic cascades has been reported in mice overexpressing $\mathrm{GH}$ (Miquet et al. 2008), the main GH signaling pathway, JAK2/ STAT5, is desensitized in liver of mice overexpressing $\mathrm{GH}$ (González et al. 2002, Miquet et al. 2004, 2005). This implies that this signaling pathway would not be involved in the proliferative effect of overexpressed $\mathrm{GH}$.

A possible explanation to justify the association between hepatocellular carcinoma and high GH levels involves the modulatory role of $\mathrm{GH}$ over the action of liver mitogens like EGF. GH has been previously described to regulate hepatic EGFR expression. The receptor expression is diminished in hypophysectomized mice, and in partially GH-deficient mutant mice, this deficiency was reversed by exogenous GH administration (Jansson et al. 1988, Johansson et al. 1989). Moreover, EGFR content was found to be increased in transgenic mice overexpressing GH (Miquet et al. 2008). The objective of this study was to analyze how GH modulates EGF signaling. For this purpose, EGF signaling pathways were analyzed in two different in vivo models: one resistant to the actions of $\mathrm{GH}$ (GHR-KO mouse); and the other with increased circulating $\mathrm{GH}$ levels (transgenic mice overexpressing bovine $\mathrm{GH}(\mathrm{bGH}))$.

\section{Materials and Methods}

\section{Reagents}

Highly purified ovine $\mathrm{GH}(\mathrm{oGH})$ of pituitary origin was obtained through the National Hormone and Pituitary Program, NIDDK, NIH, USA. Recombinant human EGF, BSA-fraction $\mathrm{V}$, and protein A Sepharose were obtained from Sigma Chemical Co.; PVDF membranes and enhanced chemiluminescence (ECL)-Plus from Amersham Biosciences. Secondary antibodies conjugated with HRP, agarose-conjugated anti-Grb2, and antibodies anti-STAT5 (C-17) and anti-EGFR (1005) were purchased from Santa Cruz Biotechnology Laboratories (Santa Cruz, CA, USA). Antibody anti-phospho-STAT5a/b Tyr694/696 was from Upstate Laboratories (Lake Placid, NY, USA). Antibodies anti-phospho-AKT Ser473, anti-AKT, anti-p44/42 MAP kinase, anti-phospho-p44/42 MAP kinase Thr202/Tyr204, anti-Grb2, anti-phospho-STAT3 Tyr705, anti-phospho-EGFR Tyr845, anti-phospho-EGFR Tyr992, and anti-phospho-EGFR Tyr1068 were from Cell Signaling Technology Inc. (Beverly, MA, USA). Antibodies anti-STAT3 and anti-SH2-containing phosphatase-2 (SHP-2) were purchased from Transduction Laboratories (Lexington, KY, USA). All other chemicals were of reagent grade.

\section{Animals}

GHR-KO mice $(-/-)$ were developed as described previously (Zhou et al. 1997). GHR-KO mice and normal littermate controls were produced by mating heterozygous $(+/-)$ carriers of the disrupted GHR/GHBP gene or homozygous knockout $(-/-)$ males with $+/-$ females. The genetic background of these animals is derived from 129/ Ola embryonic stem cells and from BALB/c, C57BL/6, and $\mathrm{C} 3 \mathrm{H}$-inbred strains. Phosphoenolpyruvate carboxykinase (PEPCK)-bGH mice containing the bGH gene fused to control sequences of the rat Pepck (Pck1 as listed in the MGI Database) gene (McGrane et al. 1990) were derived from animals kindly provided by Drs T E Wagner and J S Yun (Ohio University, Athens, OH, USA). The hemizygous transgenic mice were derived from a founder male, and were produced by mating transgenic males with normal 
C57BL/6 $\times$ C3H F1 hybrid females purchased from the Jackson Laboratory (Bar Harbor, ME, USA). Mating produced approximately equal proportion of transgenic and normal progeny. Normal siblings of transgenic mice were used as controls.

GHR-KO and PEPCK-bGH adult animals (4-7 months old) were used. The mice were housed $3-5$ per cage in a room with controlled light $(12 \mathrm{~h} \mathrm{light/day)}$ and temperature $\left(22 \pm 2{ }^{\circ} \mathrm{C}\right)$. The animals had free access to food (Lab Diet Formula 5001; PMI Inc., St Louis, MO, USA) and tap water. The appropriateness of the experimental procedure, the required number of animals used, and the method of acquisition were in compliance with federal and local laws, and with institutional regulations.

\section{Animal treatments}

GHR-KO mice, PEPCK-bGH transgenic mice, and their respective normal littermate controls were fasted for $6 \mathrm{~h}$ prior to i.p. injection with $2.5 \mathrm{mg}$ oGH per $\mathrm{kg}$ of body weight (BW) $(2.5 \mathrm{mg} / \mathrm{kg} \mathrm{BW})$ in $0.9 \% \mathrm{w} / \mathrm{v} \mathrm{NaCl}$ or with recombinant human EGF at $2 \mathrm{mg} / \mathrm{kg} \mathrm{BW}$ in $0.9 \% \mathrm{w} / \mathrm{v}$ $\mathrm{NaCl}$. Additional mice were injected with saline to evaluate basal conditions. Mice were killed $7 \cdot 5 \mathrm{~min}$ after GH injection or $10 \mathrm{~min}$ after EGF administration; the livers were removed and stored frozen at $-70{ }^{\circ} \mathrm{C}$ until liver homogenization.

\section{Preparation of liver extracts}

Liver samples were homogenized at the ratio $0 \cdot 1 \mathrm{~g}: 1 \mathrm{ml}$ in buffer composed of $1 \% \mathrm{v} / \mathrm{v}$ Triton, $0.1 \mathrm{~mol} / 1$ HEPES, $0 \cdot 1 \mathrm{~mol} / 1$ sodium pyrophosphate, $0 \cdot 1 \mathrm{~mol} / 1$ sodium fluoride, 0.01 mol/1 EDTA, 0.01 mol/1 sodium vanadate, 0.002 mol/1 phenylmethylsulfonyl fluoride, and 0.035 trypsin inhibitory units/ml aprotinin $(\mathrm{pH} 7 \cdot 4)$ at $4{ }^{\circ} \mathrm{C}$. Liver homogenates were centrifuged at $100000 \mathrm{~g}$ for $40 \mathrm{~min}$ at $4^{\circ} \mathrm{C}$ to remove insoluble material. Protein concentration of supernatants was determined by the method of Bradford (1976). An aliquot of solubilized liver was diluted in Laemmli buffer, boiled for $5 \mathrm{~min}$, and stored at $-20^{\circ} \mathrm{C}$ until electrophoresis.

\section{Immunoprecipitation}

Aliquots of solubilized liver containing $4 \mathrm{mg}$ protein were incubated at $4{ }^{\circ} \mathrm{C}$ overnight with anti-STAT5, anti-STAT3, anti-SHP-2, or agarose-conjugated anti-Grb2 antibodies. In the case of non-conjugated antibodies, after incubation, $20 \mu \mathrm{l}$ protein A Sepharose $(50 \% \mathrm{v} / \mathrm{v})$ was added to the mixture. The preparation was further incubated with constant rocking for $2 \mathrm{~h}$ and then centrifuged at $3000 \mathrm{~g}$ for $1 \mathrm{~min}$ at $4{ }^{\circ} \mathrm{C}$. The supernatant that resulted after protein immunoprecipitation was discarded, and the precipitate was washed three times with washing buffer $(0.05 \mathrm{~mol} / 1 \mathrm{Tris}, 0 \cdot 01 \mathrm{~mol} / \mathrm{l}$ vanadate, and $1 \% \mathrm{v} / \mathrm{v}$ Triton $\mathrm{X}-100, \mathrm{pH} 7 \cdot 4)$. The final pellet was resuspended in $35 \mu \mathrm{l}$ Laemmli buffer, boiled for $5 \mathrm{~min}$, and stored at $-20{ }^{\circ} \mathrm{C}$ until electrophoresis.

\section{Western blot analysis}

Samples were subjected to electrophoresis in SDS-polyacrylamide gels using Bio-Rad Mini Protean apparatus (Bio-Rad Laboratories). Electrotransference of proteins from gel to nitrocellulose PVDF membranes was performed for $1 \mathrm{~h}$ at $100 \mathrm{~V}$ (constant) using the Bio-Rad miniature transfer apparatus in $0.025 \mathrm{~mol} / \mathrm{l}$ Tris, $0.192 \mathrm{~mol} / \mathrm{l}$ glycine, and $20 \%$ $\mathrm{v} / \mathrm{v}$ methanol, $\mathrm{pH} 8 \cdot 3$. To reduce non-specific antibody binding, membranes were incubated $2 \mathrm{~h}$ at room temperature in T-TBS buffer $(0 \cdot 01 \mathrm{~mol} / 1$ Tris- $\mathrm{HCl}, 0 \cdot 150 \mathrm{~mol} / 1 \mathrm{NaCl}$, and $0 \cdot 02 \% \mathrm{v} / \mathrm{v}$ Tween $20, \mathrm{pH} 7 \cdot 6$ ), containing $3 \% \mathrm{w} / \mathrm{v}$ BSA. The membranes were then incubated overnight at $4{ }^{\circ} \mathrm{C}$ with the primary antibodies. After washing with T-TBS, the membranes were incubated with a secondary antibody conjugated with HRP for $1 \mathrm{~h}$ at room temperature and washed in T-TBS. Immunoreactive proteins were revealed by ECL-Plus (Amersham Biosciences) using preflashed Kodak XAR film (Eastman 222 Kodak). Band intensities were quantified using Gel-Pro Analyzer 4.0 software (Media Cybernetics, Silver Spring, MD, USA).

To reprobe with other antibodies, the membranes were washed with acetonitrile for $10 \mathrm{~min}$ and then incubated in stripping buffer $(2 \% \mathrm{w} / \mathrm{v}$ SDS, $0.100 \mathrm{~mol} / 1$ 2-mercaptoethanol, and $0.0625 \mathrm{~mol} / \mathrm{l}$ Tris- $\mathrm{HCl}, \mathrm{pH} 6.7$ ) for $40 \mathrm{~min}$ at $50{ }^{\circ} \mathrm{C}$ while shaking, washed with deionized water, and blocked with BSA.

\section{Statistical analysis}

Experiments were performed by analyzing all groups of animals in parallel, $n$ representing the number of different individuals used in each group. Results are presented as mean \pm s.E.M. of the number of samples indicated. Statistical analyses were performed by ANOVA followed by the Newman-Keuls multiple comparison test using the GraphPad Prism 4 statistical program by GraphPad Software, Inc. (San Diego, CA, USA). Data were considered significantly different if $P<0 \cdot 05$.

\section{Results}

Activation of EGF signaling pathways in liver of GHR-KO mice

EGFR phosphorylation at Tyr845, Tyr992, and Tyr1068 and protein content were analyzed by western blotting of liver solubilizates with specific antibodies (Fig. 1). EGFR protein levels were lower in GHR-KO mice than in normal control mice (Fig. 1A). Basal phosphorylation at Tyr845, Tyr992, and Tyr1068 was not different between normal and GHR-KO mice, but EGF-induced phosphorylation of EGFR was significantly decreased in GHR-KO mice compared to EGF-induced phosphorylation in normal mice (Fig. 1B-D and Supplementary Figure 1, see section on supplementary data given at the end of this article). When EGF-induced 

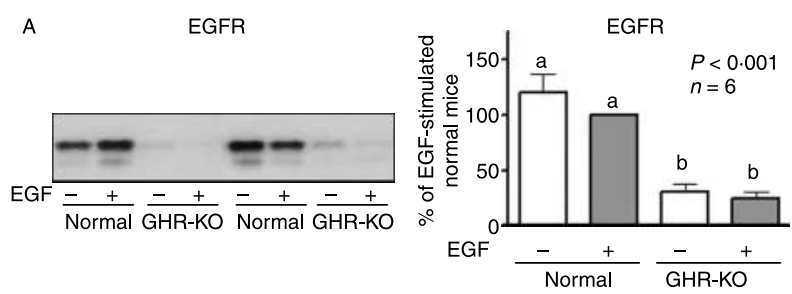

B $\quad$ PY845 EGFR

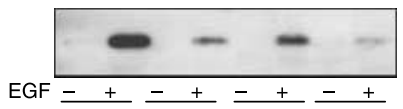

$\overline{\text { Normal }} \overline{\text { GHR-KO }} \overline{\text { Normal GHR-KO }}$

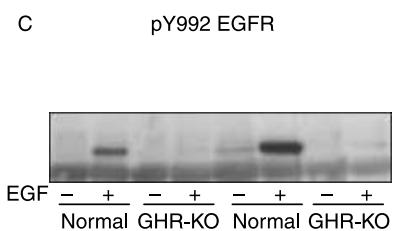

D

DY1068 EGFR
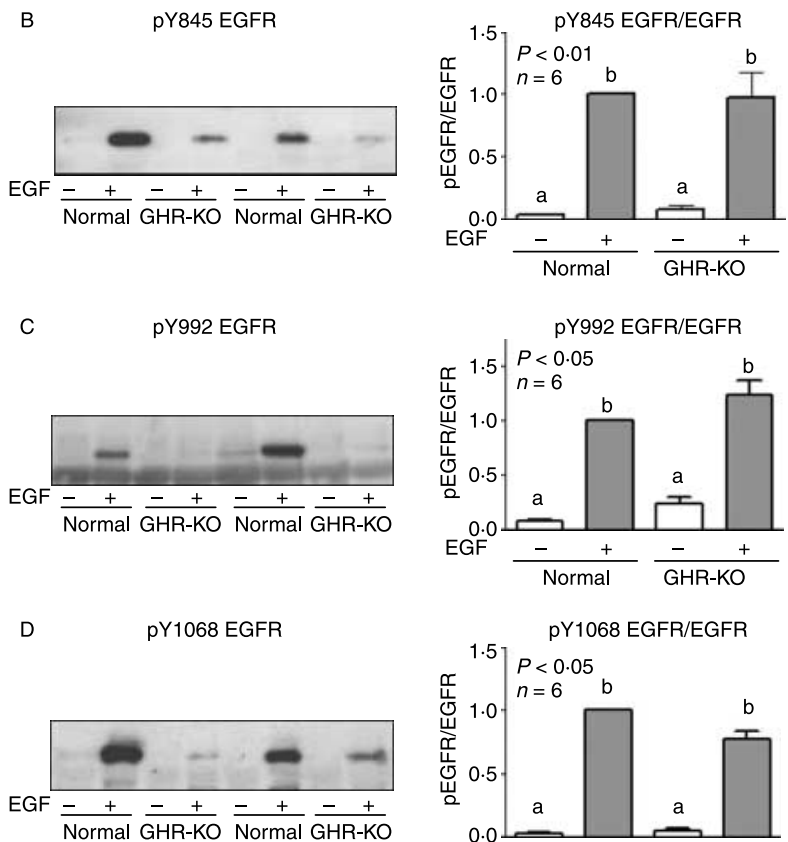

Normal GHR-KO $\overline{\text { Normal GHR-KO }}$

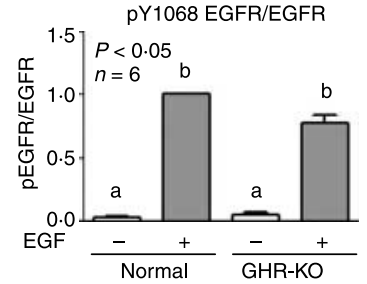

Figure 1 EGFR content and phosphorylation in normal and GHR$\mathrm{KO}$ mice. Normal and GHR-KO mice were injected i.p. with saline or EGF (2 mg/kg), killed after $10 \mathrm{~min}$, and the livers were removed. Equal amounts of solubilized liver protein were separated by SDSPAGE and subjected to immunoblot analysis. Representative results of immunoblots with anti-EGFR (A), anti-phospho-EGFR Tyr845 (B), anti-phospho-EGFR Tyr992 (C), and anti-phospho-EGFR Tyr1068 (D) are shown. Quantification was performed by scanning densitometry and expressed as percent of values measured for EGFstimulated normal mice. Data resulting form quantification analysis were used to calculate the ratio pY845, 992, 1068 EGFR/EGFR content. Data are expressed as the mean \pm s.E.M. of the indicated number of subsets $(n)$ of different individuals. Different letters denote significant difference at $P<0 \cdot 05$. Representative results from two samples per experimental condition are shown.

phosphorylation at Tyr845, Tyr992, and Tyr1068 of the receptor was correlated with the EGFR content, no significant differences were observed between EGF-induced phosphorylation of EGFR from GHR-KO and normal mice (Fig. 1).

AKT protein content and phosphorylation at the activating residue Ser473 in response to EGF stimulation were also analyzed. Neither AKT expression nor basal phosphorylation differed between GHR-KO and normal mice (Fig. 2A and Supplementary Figure 2A, see section on supplementary data given at the end of this article), but EGF-stimulated AKT phosphorylation was reduced in GHR-KO mice compared to normal mice (Fig. 2A and Supplementary Figure 2A). Similar results were observed when ERK1/2 content and phosphorylation at residues Thr202 and Tyr204 were studied. While ERK1/2 expression and basal phosphorylation did not significantly differ between GHR-KO and normal mice, EGF activation of ERK1/2 was significantly diminished in GHR-KO mice (Fig. 2B and Supplementary Figure 2B).

Similarly, EGF signaling through STAT3 and STAT5 was analyzed in GHR-KO and normal mice. As expected in the context of an extended decrease of EGF signaling in GHR-KO mice, STAT3 and STAT5 phosphorylation due to EGF stimulus was diminished in GHR-KO mice without any changes in protein content and basal phosphorylation between normal and GHR-KO mice (Fig. 2C, D, and Supplementary Figure 2C and D).

\section{Activation of EGF and GH signaling pathways in $G H$ transgenic mice}

EGF signaling was subsequently analyzed in a mouse model of $\mathrm{GH}$ overexpression. As already mentioned earlier, transgenic mice overexpressing $\mathrm{GH}$ display higher EGFR expression and increased basal phosphorylation at residue Tyr845 (Fig. 3A and B; Miquet et al. 2008). Basal phosphorylation at residues Tyr992 and Tyr1068 of the EGFR is also increased in these mice (Fig. 3C, D, and Supplementary Figure 3, see section on supplementary data given at the end of this article). However, in transgenic animals, EGF-induced phosphorylation of EGFR was found to be similar to the corresponding EGF-stimulated control siblings (Fig. 3A-C, and Supplementary Figure 3). When phosphorylation of the EGFR at the different tyrosine residues was correlated with EGFR content, this analysis showed that EGF-induced response of the receptor is diminished in transgenic animals compared with normal mice (Fig. 3). It was reported that GH induces EGFR phosphorylation at Tyr1068, Tyr845, and Tyr992 in liver (Yamauchi et al. 1998, Kim et al. 1999, Huang et al. 2003). Nevertheless, in our experimental conditions, we were not able to detect EGFR phosphorylation after acute $\mathrm{GH}$ stimulation in liver of normal or transgenic mice (Fig. 3A-C).

AKT and ERK1/2 protein contents and phosphorylation were also analyzed in liver homogenates from normal and transgenic mice stimulated with EGF, GH, or saline. AKT protein content as well as its basal phosphorylation was increased in PEPCK-bGH transgenic mice (Fig. 4A and Supplementary Figure 4A, see section on supplementary data given at the end of this article), while for ERK1/2, its expression was increased in transgenic mice compared to normal siblings (Fig. 4B and Supplementary Figure 4B; Miquet et al. 2008), but not its basal phosphorylation (Fig. 4B and Supplementary Figure 4B). EGF stimulation caused similar levels of AKT and ERK1/2 phosphorylation in normal and transgenic mice (Fig. 4A, B and Supplementary Figure $4 \mathrm{~A}$ and $\mathrm{B}$ ); however, in coincidence with results 

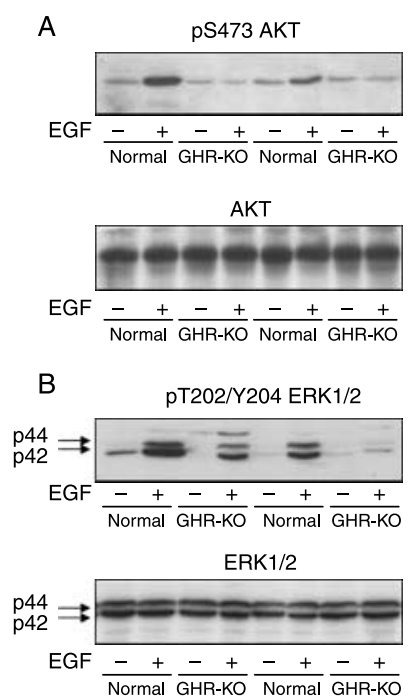
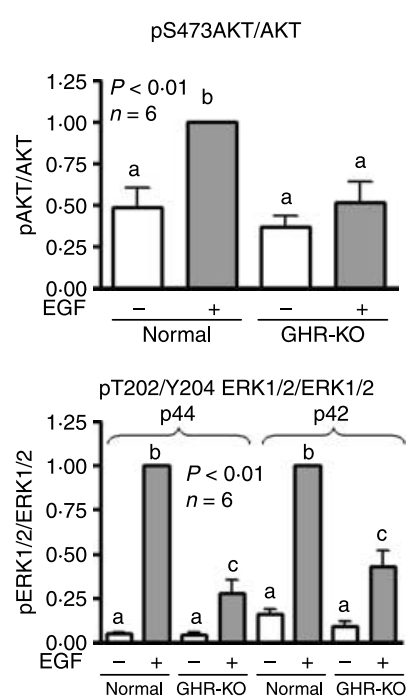

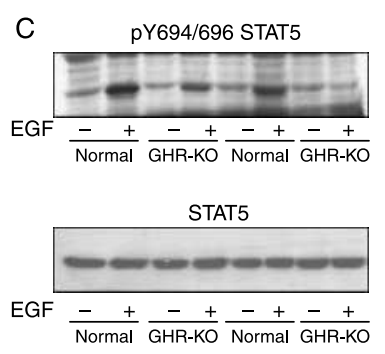

D

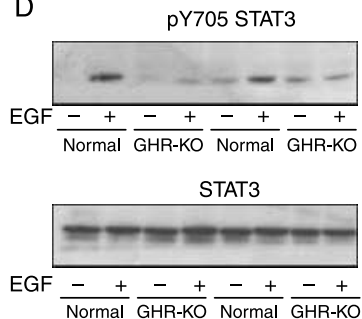

pY694/696 STAT5/STAT5

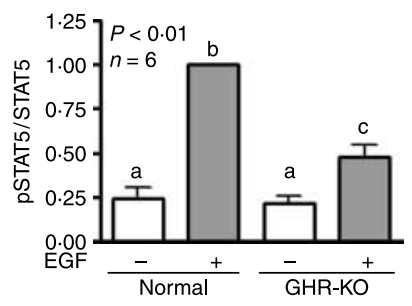

PY705 STAT3/STAT3

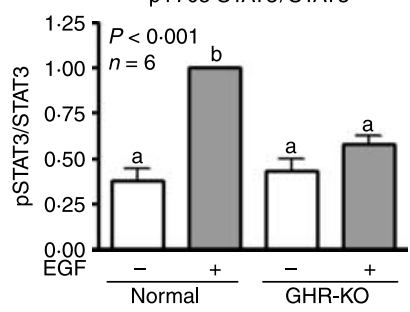

Figure 2 AKT, ERK1/2, STAT5, and STAT3 content and phosphorylation in normal and GHR-KO mice. Normal and GHR-KO mice were injected i.p. with saline or EGF $(2 \mathrm{mg} / \mathrm{kg})$, killed after $10 \mathrm{~min}$, and the livers were removed. Equal amounts of solubilized liver protein were separated by SDS-PAGE and subjected to immunoblot analysis. Representative results of immunoblots with anti-phospho-AKT Ser473 and anti-AKT (A), anti-phospho-p44/42 MAP kinase Thr202/Tyr204 and anti-p44/42 MAP kinase (B), anti-phospho-STAT5a/b Tyr694/696 and anti-STAT5 (C), and anti-phospho-STAT3 Tyr705 and anti-STAT3 (D) are shown. Quantification was performed by scanning densitometry and expressed as percent of values measured for EGF-stimulated normal mice. Data resulting form quantification analysis were used to calculate the ratio between the rate of phosphorylated protein and the protein content. Data are expressed as the mean \pm S.E.M. of the indicated number of subsets $(n)$ of different individuals. Different letters denote significant difference at $P<0 \cdot 01$. Representative results from two samples per experimental condition are shown.

obtained for EGFR, EGF-induced phosphorylation of AKT and ERK1/2 related to their protein content demonstrated that AKT and ERK1/2 response is diminished in the transgenic mice (Fig. 4A and B). On the other hand, GH did not significantly induce AKT and ERK1/2 phosphorylation in normal and transgenic mice liver (Fig. 4A and B; Miquet et al. 2008).

Subsequently, STAT3 and STAT5 activation by EGF was analyzed in normal and transgenic mice and compared with activation of these transcription factors by GH. In contrast to results observed for AKT and ERK1/2 phosphorylation, STAT3 and STAT5 activation was not significantly induced by EGF in transgenic mice (Fig. 4C, D, and Supplementary Figure $4 \mathrm{C}$ and $\mathrm{D}$ ), resembling previously described desensitization of GH signal through these proteins (González et al. 2002, Miquet et al. 2004, 2005, 2008). STAT3 and STAT5 protein contents were similar in normal and transgenic animals (Fig. 4C, D, and Supplementary Figure 4C and D). In order to investigate a possible mechanism involved in STAT3 and STAT5 desensitization upon EGF stimulus, we performed immunoprecipitation assays to analyze STAT association to the EGFR. For this purpose, STAT3 and STAT5 were immunoprecipitated from solubilized liver, and associated EGFR was determined by western blotting of immunoprecipitated samples. As shown in Fig. 5, association of EGFR to STAT3 and STAT5 was significantly diminished in transgenic mice (Fig. 5A and B). As expected from previous results (Fig. 4C and D), immunoprecipitated STAT proteins levels were similar between normal and transgenic mice livers (Fig. 5A and B).

The adaptor protein GRB2 plays a critical role in coupling signal from EGFR with Ras/MAPK pathway. GRB2 has been demonstrated to negatively regulate STAT3 activation by the EGF. The proposed mechanism involved in such desensitization implies GRB2 binding to the EGFR, inhibiting the interaction between STAT3 and EGFR by competitive binding to the same region of the receptor (Zhang et al. 2003). Because we observed inhibition of EGFinduced activation of STAT3 in the transgenic mice overexpressing GH, we studied whether GRB2 expression was increased in the transgenic mice, as this could be involved in STAT3 desensitization. However, no significant differences in the expression of this protein were detected between normal and transgenic mice (Fig. 6A). Alternatively, association of GRB2 to EGFR could be increased in the transgenic mice. To analyze this possibility, co-immunoprecipitation of EGFR with GRB2 was determined. The association of GRB2 to the EGFR was not augmented in the transgenic mice as shown in Fig. 6B.

Previous results from our laboratory have demonstrated that the tyrosine SHP-2 content was increased in liver microsomes from transgenic animals, while the abundance of this protein in whole solubilized liver was the same in normal and transgenic mice (Miquet et al. 2004). SHP-2 has 

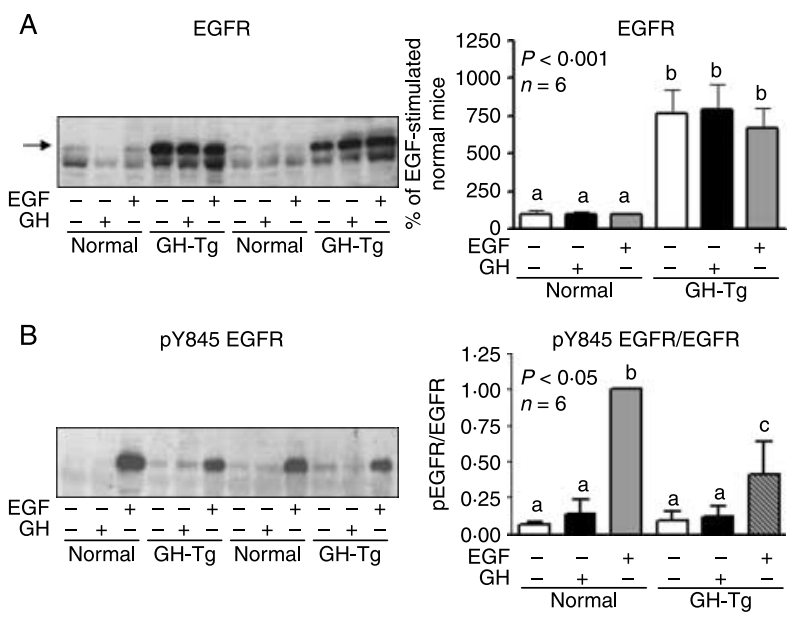

C pY992 EGFR
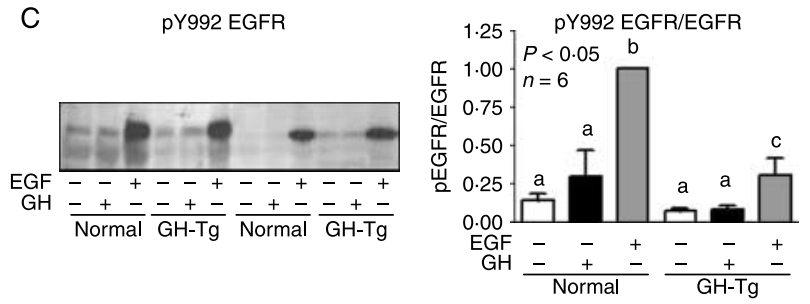

D pY1068 EGFR
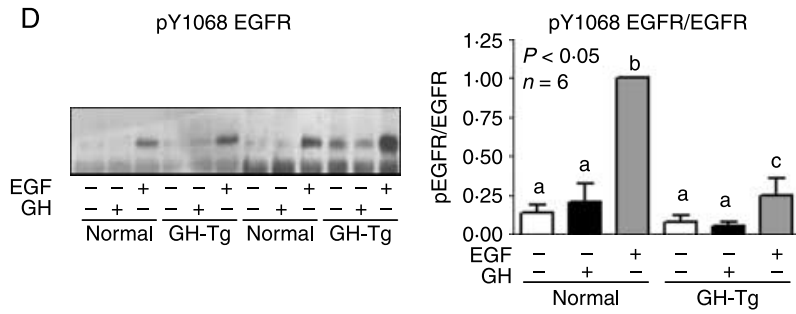

Figure 3 EGFR content and phosphorylation in normal and $\mathrm{GH}$-overexpressing mice. Normal and PEPCK-bGH transgenic mice (GH-Tg) were injected i.p. with saline, $\mathrm{GH}(2.5 \mathrm{mg} / \mathrm{kg} \mathrm{BW})$, or EGF (2 mg/kg BW), killed after 7.5 or 10 min respectively, and the livers were removed. Equal amounts of solubilized liver protein were separated by SDS-PAGE and subjected to immunoblot analysis. Representative results of immunoblots with anti-EGFR (A), anti-phospho-EGFR Tyr845 (B), anti-phospho-EGFR Tyr992 (C), and anti-phospho-EGFR Tyr1068 (D) are shown. Quantification was performed by scanning densitometry and expressed as percent of values measured for EGF-stimulated normal mice. Data resulting form quantification analysis were used to calculate the ratio pY845, 992, 1068 EGFR/EGFR content. Data are expressed as the mean \pm S.E.M. of the indicated number of subsets $(n)$ of different individuals. Different letters denote significant difference at $P<0 \cdot 05$. Representative results from two samples per experimental condition are shown.

been implicated in the negative regulation of $\mathrm{GH}$ signaling to STAT5 (Stofega et al. 2000), and association between SHP-2 and STAT5 has previously been described in hematopoietic and mammary cells (Chughtai et al. 2002, Chen et al. 2004). Increased SHP-2 association of STAT5 in transgenic mice liver could lead to inactivation of the transcription factor. To test this hypothesis, we analyzed co-immunoprecipitation of SHP-2 with STAT5 and observed a significant increase in the basal association of SHP-2 and STAT5 in transgenic mice liver, which did not vary upon hormone stimulation (Fig. 6C).

\section{Discussion}

Crosstalk between GH and EGF signaling pathways comprising diverse signaling mediators has been described, while EGFR expression has been demonstrated to be regulated by GH (Jansson et al. 1988, Johansson et al. 1989). Partially, GH-deficient mutant mice and hypophysectomized mice showed reduced expression of the EGFR in liver. Moreover, $\mathrm{GH}$ administration to these mice induced the expression of the receptor approaching EGFR levels found in normal mice (Johansson et al. 1989). GH has also been demonstrated to induce EGFR phosphorylation at tyrosines 845, 992, 1068, and 1173 (Yamauchi et al. 1997, 1998, Kim et al. 1999, Huang et al. 2003). Another level of interaction between GH and EGF signaling involves GH-induced EGFR phosphorylation at threonine residues (Huang et al. 2003). Such phosphorylation reduces EGF-induced EGFR degradation, thus modulating EGFR trafficking and signaling (Huang et al. 2003).

Considering the evidence implying EGF or EGFR overexpression and/or hyperactivation of the receptor with the development of liver cancer (Normanno et al. 2006), given that numerous studies have demonstrated an important association between high GH levels and development of hepatocarcinoma (Snibson 2002, Bartke 2003) and taking into account that $\mathrm{GH}$ and EGF share many signaling pathways as well as the control of expression and activation of proteins involved in the modulation of the signal, the aim of this study was to analyze GH modulatory effects on EGF signaling in the liver. Our hypothesis was that high GH levels would result in the exacerbation of EGF signaling, leading to augmented cell proliferation, while loss of $\mathrm{GH}$ action would render decreased EGF signaling. EGF signal transduction pathways were then studied in two different in vivo models: GHR-KO mice, in which $\mathrm{GH}$ action is abolished, and $\mathrm{GH}$ transgenic mice, a model of high GH levels.

EGFR was decreased in GHR-KO mice, which agrees with previous results obtained in hypophysectomized and partially GH-deficient, genetic mutant 'little' (lit/lit) mice (Jansson et al. 1988, Johansson et al. 1989). Consistent with diminished EGFR expression in GHR-KO mice, EGFinduced phosphorylation of the receptor at tyrosines 845 , 992, and 1068 was also decreased (Fig. 1 and Supplementary Figure 1). Correlation between phosphorylation and protein content indicates that the diminished response of the receptor can be attributed to its down-regulation (Fig. 1).

Activation of the different signaling pathways triggered by EGF was also diminished or suppressed in GHR-KO mice. EGF-induced hepatic STAT5 and ERK1/2 phosphorylation was reduced in GHR-KO mice, while EGF did not activate STAT3 and AKT in these animals (Fig. 2). These results are in 
A

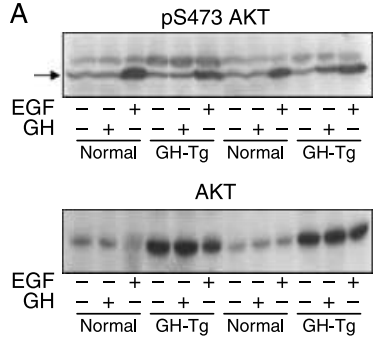

B

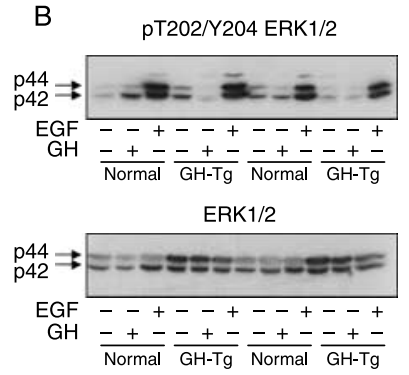

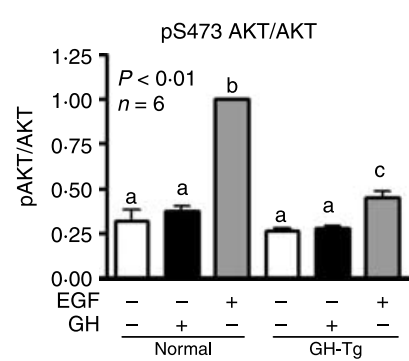

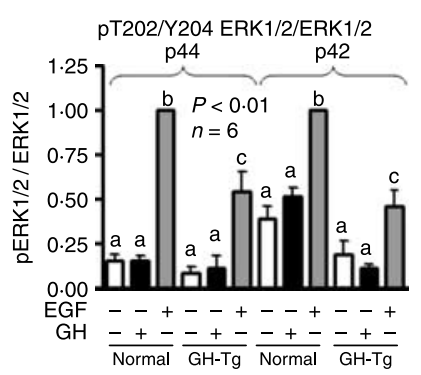

C

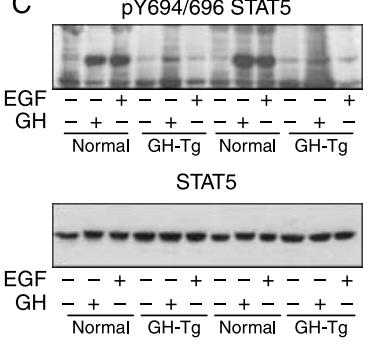

$\mathrm{D}$

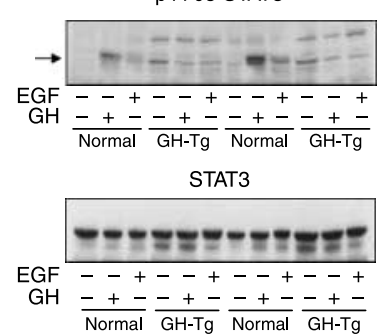

pY694/696 STAT5/STAT5

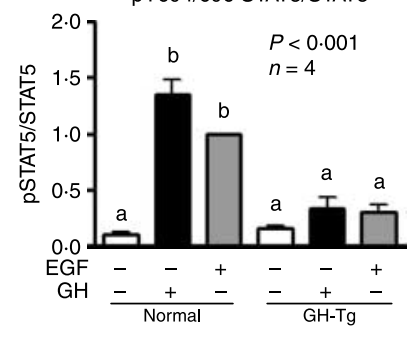

pY705 STAT3/STAT3

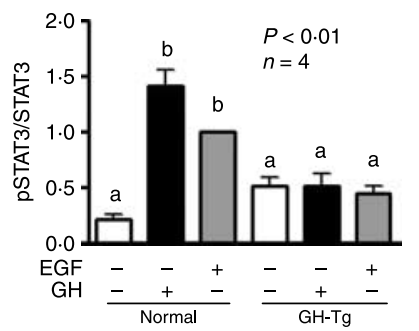

Figure 4 AKT, ERK1/2, STAT5, and STAT3 content and phosphorylation in normal and GH-overexpressing mice. Normal and PEPCK-bGH transgenic mice (GH-Tg) were injected i.p. with saline, GH (2.5 mg/kg BW), or EGF (2 mg/kg BW), killed after 7.5 or 10 min respectively, and the livers were removed. Equal amounts of solubilized liver protein were separated by SDS-PAGE and subjected to immunoblot analysis. Representative results of immunoblots with anti-phospho-AKT Ser473 and anti-AKT (A), anti-phospho-p44/42 MAP kinase Thr202/Tyr204 and anti-p44/42 MAP kinase (B), anti-phospho-STAT5a/b Tyr694/696 and anti-STAT5 (C), and anti-phospho-STAT3 Tyr705 and anti-STAT3 (D) are shown. Quantification was performed by scanning densitometry and expressed as percent of values measured for EGF-stimulated normal mice. Data resulting form quantification analysis were used to calculate the ratio between the rate of phosphorylated protein and the protein content. Data are expressed as the mean \pm S.E.M. of the indicated number of subsets $(n)$ of different individuals. Different letters denote significant difference at $P<0 \cdot 01$. Representative results from two samples per experimental condition are shown.

accordance with the hypothesis of diminished EGF signaling in a context of suppressed GH action. However, certain signals are only reduced, while others cannot be induced by the exogenous EGF stimulus. Such discrepancy could be attributed to the different degree of activation of the several signaling pathways in response to EGF. Indeed, EGF induction of STAT5 and ERK1/2 was more pronounced than the activation of STAT3 and AKT in normal mice (Fig. 2). In summary, decreased EGFR levels lead to reduced signaling, evidenced by a reduction of the activation of potently induced molecules such as STAT5 and ERK1/2, whereas for moderately stimulated proteins, such as STAT3 and AKT, the signal is inhibited.

Abrogation of GH action and diminished levels of the hormone have been associated with life extension and delayed occurrence of fatal neoplastic disease in different mouse mutants (Anisimov 2001, Ikeno et al. 2003). Moreover, several studies have demonstrated that GH-deficient rodents are more resistant than their normal siblings for development of different types of tumors in response to carcinogen administration (Styles et al. 1990, Pollak et al. 2001, Swanson \& Unterman 2002). Results from our current study show that blunted GH action is associated with reduced expression of the EGFR and, concomitantly, reduced EGF signaling. Considering the pro-proliferative actions of EGF, the outcomes suggest that $\mathrm{GH}$ receptor and $\mathrm{GH}$ signaling mediators could be targets of anti-tumoral therapies, perhaps in association with EGFR inhibitors or EGF antagonists. In this context, one could envision that increased levels of GH would hypersensitize EGF signaling and promote cell proliferation. To test this hypothesis, EGF signaling was analyzed in transgenic mice overexpressing $\mathrm{GH}$.

EGFR content has been shown to be increased in transgenic mice overexpressing GH (Miquet et al. 2008), which was corroborated in this study. Considering the increase in EGFR expression, EGF-induced EGFR phosphorylation relative to protein content was found to be diminished in the transgenic animals (Fig. 3). Analysis of the signaling molecules ERK1/2 and AKT, which have been extensively associated with proliferative and survival cellular events, revealed that they were overexpressed in the transgenic mice (Fig. 4). Consistent with results obtained for the EGFR, EGF-induced phosphorylation of AKT and ERK1/2 relative to protein content was also diminished (Fig. 4). EGF activation of STAT3 and STAT5 was not significantly induced in transgenic mice (Fig. 4), resembling previously described desensitization of GH signal through these proteins in GH-overexpressing mice (González et al. 2002, Miquet et al. 2004, 2008). In conclusion, overexpression of GH induces the increase in EGFR expression but does not result in up-regulation of EGF signaling. Indeed, the effects of high GH levels differ depending on the signaling 
A

WB: EGFR

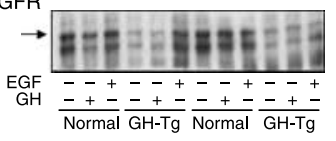

WB: STAT5

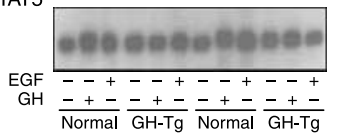

B

IP: STAT3

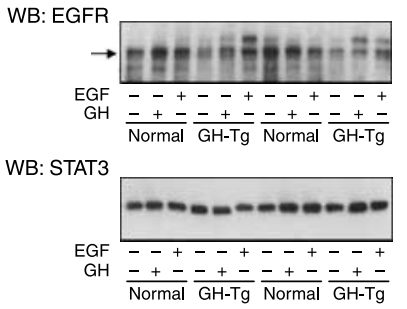

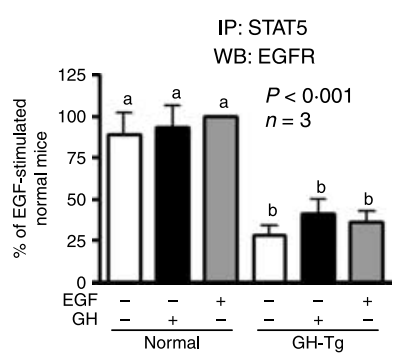

IP: STAT3

WB: EGFR

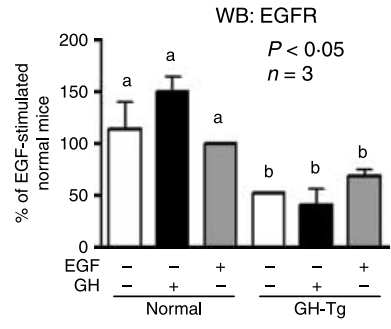

Figure 5 EGFR co-immunoprecipitation with STAT5 and STAT3 in normal and $\mathrm{GH}$-overexpressing transgenic mice. Normal and PEPCK-bGH transgenic mice (GH-Tg) were injected i.p. with saline, GH $(2.5 \mathrm{mg} / \mathrm{kg} \mathrm{BW})$, or EGF (2 mg/kg BW), killed after 7.5 or 10 min respectively, and the livers were removed. Equal amounts of solubilized liver protein were immunoprecipitated with anti-STAT5 or anti-STAT3 antibodies. Samples were separated by SDS-PAGE and subjected to immunoblot analysis. Representative results of immunoblots with anti-EGFR or immnuprecipitating antibodies (A and B) are shown. Quantification was performed by scanning densitometry and expressed as percent of values measured for EGF-stimulated normal mice. Data are the mean \pm s.E.M. of the indicated number of subsets $(n)$ of different individuals. Different letters denote significant difference at $P<0 \cdot 05$. Representative results from two samples per experimental condition are shown.

pathway analyzed: AKT and ERK1/2 pathways show diminished activation, while STAT3 and STAT5 activation is abrogated. These observations suggest that differential mechanisms and control systems are involved in $\mathrm{GH}$ modulation of EGF signaling. Selective desensitization of EGF signaling through the STATs has been previously reported in a different cellular and hormonal context, breast cancer cells overexpressing the estrogen receptor (Boerner et al. 2005).

The activation of the STATs is highly regulated but incompletely understood. GRB2 and suppressors of cytokine signaling (SOCS) can inhibit STAT-mediated EGFR signaling. GRB2 inhibits STAT3 activation by binding to the STAT-docking site on the EGFR, while SOCS3 inhibits STAT3 phosphorylation by binding to JAK (Endo et al. 1997, Quesnelle et al. 2007). To investigate possible intracellular mechanisms triggered by $\mathrm{GH}$ which specifically act on STATs signaling, STAT3 and STAT5 association to EGFR was analyzed in solubilized liver from normal and transgenic mice. Diminished association between EGFR and the STATs was found in the transgenic mice (Fig. 5), which suggests that the mechanisms involved in STATs desensitization to EGF stimulation may implicate inhibition of recruitment of the
STATs to activated EGFR. Regarding SOCS3 involvement in STATs desensitization, we have previously described that expression of this SOCS protein is diminished in GH-overexpressing mice (González et al. 2002, Miquet et al. 2004). Decreased SOCS3 expression could probably be involved in the increased basal tyrosine phosphorylation of STAT3 observed in these transgenic mice but cannot explain STAT3 insensitivity to EGF stimulus. However, GRB2 overexpression could be involved in STAT3 desensitization upon EGF stimulus. Following EGF stimulation, GRB2 directly binds to the cytoplasmic domain of the EGFR at phosphorylated residues Tyr1068 and Tyr1086 to initiate the
A

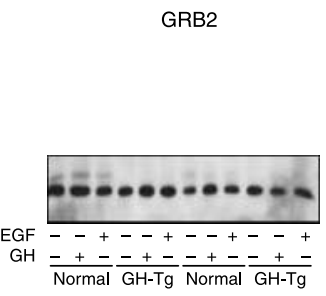

B

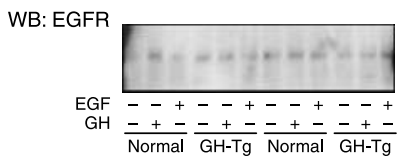

WB: Grb2

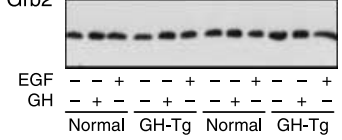

C

WB: SHP-2

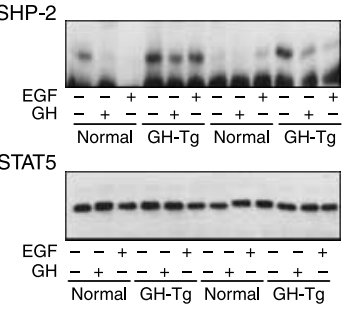

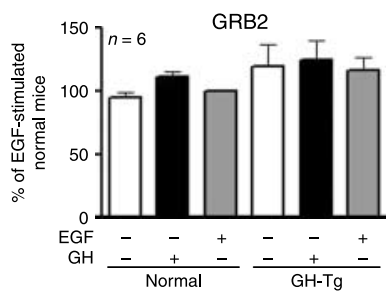

IP: GRB2 WB: EGFR

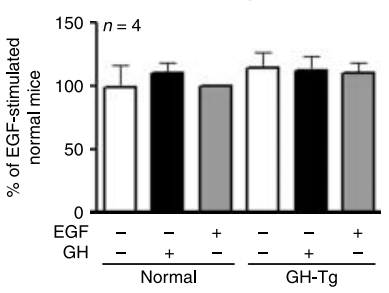

IP: STAT5 WB: SHP-2

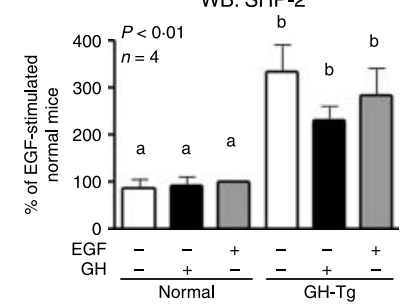

Figure 6 GRB2 content, GRB2/EGFR co-immunoprecipitation, and STAT5/SHP-2 co-immunoprecipitation in normal and $\mathrm{GH}$-overexpressing transgenic mice. Normal and PEPCK-bGH transgenic mice $(\mathrm{GH}-\mathrm{Tg})$ were injected i.p. with saline, $\mathrm{GH}(2.5 \mathrm{mg} / \mathrm{kg} \mathrm{BW})$, or EGF (2 mg/kg BW), killed after 7.5 or 10 min respectively, and the livers were removed. Equal amounts of solubilized liver protein were separated by SDS-PAGE and subjected to immunoblot analysis with anti-Grb2 (A) or were immunoprecipitated with anti-Grb2 or anti-STAT5 antibodies, separated by SDS-PAGE and subjected to immunoblot analysis with anti-EGFR and anti-Grb2 (B) or anti-SHP2 and anti-STAT5 (C) respectively. Representative results of immunoblots are shown. The amount of immunoprecipitated protein was determined by immunoblotting with the corresponding antibody. Quantification was performed by scanning densitometry and expressed as percent of values measured for EGF-stimulated normal mice. Data are the mean \pm S.E.M. of the indicated number of subsets $(n)$ of different individuals. Different letters denote significant difference at $P<0 \cdot 05$. Representative results from two samples per experimental condition are shown. 
activation of the Ras/MAPK pathway (Batzer et al. 1994). Multiple tyrosine residues, including Tyr1068 and Tyr1086, were also reported to be required for receptor binding and activation of STAT3 in EGF signaling (Coffer \& Kruijer 1995). The mechanism involved in the negative regulation of STAT3 by GRB2 implies competitive binding to the EGFR. We evaluated whether desensitization of EGF-induced STAT3 phosphorylation could be associated with GRB2 up-regulation and/or increased association of Grb2 to the EGFR in the transgenic mice overexpressing GH, but no significant differences in GRB2 protein content or GRB2 association to EGFR between normal and transgenic mice were found (Fig. 6A and B).

SHP-2 is a Src homology 2 domain-containing PTP involved in signal transduction of a variety of cytokines and growth factors. SHP-2 plays a negative role in certain intracellular processes, while in others the phosphatase enhances the signaling, thus having dual functions (Chong \& Maiese 2007). SHP-2 has been described to negatively regulate GH signaling to STAT5 activation (Stofega et al. 2000). The phosphatase was described to be significantly increased in liver membranes from transgenic mice overexpressing $\mathrm{GH}$, while the abundance of this protein in solubilized liver was the same in normal and transgenic mice (Miquet et al. 2004). Considering studies describing association between SHP-2 and STAT5 in models other than liver (Chughtai et al. 2002, Chen et al. 2004), we analyzed co-immunoprecipitation of SHP-2 with STAT3 and STAT5 in liver from normal and transgenic mice. Our results demonstrate that SHP-2 associates with STAT5 independently of exogenous stimulation. This association is increased in $\mathrm{GH}$-overexpressing transgenic animals (Fig. 6C). Increased recruitment of SHP-2 to liver membranes induced by overexpressed GH (Miquet et al. 2004) might sequestrate and inhibit STAT5 activation by growth factors, EGF among them. However, the proposed mechanism cannot explain STAT3 desensitization as we were not able to detect association between SHP-2 and STAT3 in the liver.

In conclusion, modulation of EGF signaling by GH is not directly correlated to EGFR expression. While abrogation of GH biological action results in decreased EGFR expression and a concomitant decrease in EGF signaling, high circulating levels of GH induce EGFR overexpression but this is not associated with an increase of the response to an acute EGF stimulus. Moreover, effects triggered by high GH levels differ depending on the signaling pathway analyzed: AKT and ERK1/2 are activated - albeit to a minor extent - by the exogenous EGF stimulus in normal and transgenic mice, while STAT3 and STAT5 responses to administrated EGF are abrogated.

This study provides evidence not only for the crosstalk between GH and EGF signaling in the liver but also for different mechanisms controlling EGF-induced signaling pathways. Inhibitory effects of high GH levels on the STATs appear not to be the result of general inhibition of EGF signaling since ERK1/2 and AKT were activated following EGF stimulation in this mouse model. EGFR-, ERK1/2-, and AKT-diminished phosphorylation relative to protein content could be due to overexpression of nonfunctional protein or to other compensatory mechanisms that may be triggered by $\mathrm{GH}$ overexpression, which could account for attenuation of EGFR signaling through AKT and ERK1/2; further studies will be needed to unravel these observations. Increased association between SHP-2 and STAT5 could contribute to the observed effects of overexpressed GH over STAT5-mediated EGF signaling. Considering that the STATs have been proposed as targets for therapeutic regimens for several cancers (Yu \& Jove 2004) and given that dominant-negative forms of STAT5 have been demonstrated to prevent tumor growth in xenograft studies (Yamashita et al. 2003), modulation of STATs may provide a unique opportunity for anti-tumor-targeted therapy (Quesnelle et al. 2007). A combination of therapeutic agents acting on both GH and EGF signaling is also a possibility but, before developing this approach, it is essential to extensively dissect the crosstalk between GH and EGF signaling.

\section{Supplementary data}

This is linked to the online version of the paper at http://dx.doi.org/10.1677/ JOE-09-0372.

\section{Declaration of interest}

The authors declare that there is no conflict of interest that would prejudice the impartiality of this scientific work.

\section{Funding}

LG, JGM, AIS, FPD, and DT are Career Investigators of CONICET, and MED is supported by a Fellowship from UBA. Support for these studies was provided by UBA, CONICET, and ANPCYT (Argentina) to DT and LG, and by NIH via grant AG 19899 and by the Ellison Medical Foundation to $\mathrm{AB}$.

\section{References}

Anisimov VN 2001 Mutant and genetically modified mice as models for studying the relationship between aging and carcinogenesis. Mechanisms of Ageing and Development 122 1221-1255.

Barreca A, Voci A, Minuto F, de Marchis M, Cecchelli E, Fugassa E, Giordano G \& Gallo G 1992 Effect of epidermal growth factor on insulin-like growth factor-I (IGF-I) and IGF-binding protein synthesis by adult rat hepatocytes. Molecular and Cellular Endocrinology 84 119-126.

Bartke A 2003 Can growth hormone (GH) accelerate aging? Evidence from GH-transgenic mice Neuroendocrinology 78 210-216.

Batzer AG, Rotin D, Ureña JM, Skolnik EY \& Schlessinger J 1994 Hierarchy of binding sites for Grb2 and Shc on the epidermal growth factor receptor. Molecular and Cellular Biology 14 5192-5201.

Boeri Erba E, Bergatto E, Cabodi S, Silengo L, Tarone G, Defilippi P \& Jensen ON 2005 Systematic analysis of the epidermal growth factor receptor by mass spectrometry reveals stimulation-dependent multisite phosphorylation. Molecular and Cellular Proteomics 4 1107-1121. 
Boerner JL, Gibson MA, Fox EM, Posner ED, Parsons SJ, Silva CM \& Shupnik MA 2005 Estrogen negatively regulates epidermal growth factor (EGF)-mediated signal transducer and activator of transcription 5 signaling in human EGF family receptor-overexpressing breast cancer cells. Molecular Endocrinology 19 2660-2670.

Bradford MM 1976 A rapid and sensitive method for the quantitation of microgram quantities of protein utilizing the principle of protein-dye binding. Analytical Biochemistry 72 248-254.

Brooks AJ, Wooh JW, Tunny KA \& Waters MJ 2008 Growth hormone receptor; mechanism of action. International Journal of Biochemistry \& Cell Biology 40 1984-1989.

Chen J, Yu W-M, Bunting KD \& Qu C-K 2004 A negative role of SHP-2 tyrosine phosphatase in growth factor-dependent hematopoietic cell survival. Oncogene 23 3659-3669.

Chong ZZ \& Maiese K 2007 The src homology 2 domain tyrosine phosphatase SHP-1 and SHP-2: diversified control of cell growth, inflammation, and injury. Histology and Histopathology 22 1251-1267.

Chughtai N, Schimchowitsch S, Lebrun J-J \& Ali S 2002 Prolactin induces SHP-2 association with Stat5, nuclear translocation, and binding to the $\beta$-casein gene promoter in mammary cells. Journal of Biological Chemistry 277 31107-31114.

Coffer PJ \& Kruijer W 1995 EGF receptor deletions define a region specifically mediating STAT transcription factor activation. Biochemical and Biophysical Research Communications 210 74-81.

Endo TA, Masuhara M, Yokouchi M, Suzuki R, Sakamoto H, Mitsui K, Matsumoto A, Tanimura S, Ohtsubo M, Misawa H et al. 1997 A new protein containing an SH2 domain that inhibits JAK kinases. Nature 387 921-924.

González L, Miquet JG, Sotelo AI, Bartke A \& Turyn D 2002 Cytokineinducible $\mathrm{SH} 2$ protein up-regulation is associated with desensitization of GH signaling in GHRH-transgenic mice. Endocrinology 143 386-394.

Grunnet N, Peng X \& Tygstrup N 1999 Growth factors and gene expression in cultured rat hepatocytes. Journal of Hepatology 31 117-122.

Hartmann H, Schmitz F, Christ B, Jungermann K \& Creutzfeldt W 1990 Metabolic actions of insulin-like growth factor-I in cultured hepatocytes from adult rats. Hepatology 12 1139-1143.

Henson ES \& Gibson SB 2006 Surviving cell death through epidermal growth factor (EGF) signal transduction pathways: implications for cancer therapy. Cellular Signalling 18 2089-2097.

Huang Y, Kim SO, Jiang J \& Frank SJ 2003 Growth hormone-induced phosphorylation of epidermal growth factor (EGF) receptor in 3T3-F442A cells. Modulation of EGF-induced trafficking and signaling. Journal of Biological Chemistry 278 18902-18913.

Huang Y, Chang Y, Wang X, Jiang J \& Frank SJ 2004 Growth hormone alters epidermal growth factor receptor binding affinity via activation of extracellular signal-regulated kinases in 3T3-F442A cells. Endocrinology 145 3297-3306.

Ikeno Y, Bronson RT, Hubbard GB, Lee S \& Bartke A 2003 Delayed occurrence of fatal neoplastic diseases in ames dwarf mice: correlation to extended longevity. Journals of Gerontology. Series A, Biological Sciences and Medical Sciences 58 291-296.

Ito Y, Takeda T, Sakon M, Tsujimoto M, Higashiyama S, Noda K, Miyoshi E, Monden M \& Matsuura N 2001 Expression and clinical significance of erb-B receptor family in hepatocellular carcinoma. British Journal of Cancer 84 1377-1383.

Jansson JO, Ekberg S, Hoath SB, Beamer WG \& Frohman LA 1988 Growth hormone enhances hepatic epidermal growth factor receptor concentration in mice. Journal of Clinical Investigation 82 1871-1876.

Jenkins PJ 2004 Acromegaly and cancer. Hormone Research 62 108-115.

Johansson S, Husman B, Norstedt G \& Andersson G 1989 Growth hormone regulates the rodent hepatic epidermal growth factor receptor at a pretranslational level. Journal of Molecular Endocrinology 3 113-120.

Jorissen RN, Walker F, Pouliot N, Garrett TP, Ward CW \& Burgess AW 2003 Epidermal growth factor receptor: mechanisms of activation and signalling. Experimental Cell Research 284 31-53.

Kim SO, Houtman JC, Jiang J, Ruppert JM, Bertics PJ \& Frank SJ 1999 Growth hormone-induced alteration in ErbB-2 phosphorylation status in 3T3-F442A fibroblasts. Journal of Biological Chemistry 274 36015-36024.
Kimura M \& Ogihara M 1998 Effects of insulin-like growth factor I and II on DNA synthesis and proliferation in primary cultures of adult rat hepatocytes. European Journal of Pharmacology 354 271-281.

Lanning NJ \& Carter-Su C 2007 Recent advances in growth hormone signaling. Reviews in Endocrine \& Metabolic Disorders 7 225-235.

McGrane MM, Yun JS, Moorman AF, Lamers WH, Hendrick GK, Arafah BM, Park EA, Wagner TE \& Hanson RW 1990 Metabolic effects of developmental, tissue-, and cell-specific expression of a chimeric phosphoenolpyruvate carboxykinase (GTP)/bovine growth hormone gene in transgenic mice. Journal of Biological Chemistry 265 22371-22379.

Miquet JG, Sotelo AI, Bartke A \& Turyn D 2004 Suppression of growth hormone (GH) Janus tyrosine kinase 2/signal transducer and activator of transcription 5 signaling pathway in transgenic mice overexpressing bovine GH. Endocrinology 145 2824-2832.

Miquet JG, Sotelo AI, Bartke A \& Turyn D 2005 Desensitization of the JAK2/STAT5 GH signaling pathway associated with increased CIS protein content in liver of pregnant mice. American Journal of Physiology. Endocrinology and Metabolism 289 E600-E607.

Miquet JG, González L, Matos MN, Hansen C, Louis A, Bartke A, Turyn D \& Sotelo AI 2008 Transgenic mice overexpressing GH exhibit hepatic upregulation of GH-signaling mediators involved in cell proliferation. Journal of Endocrinology 198 317-330.

Normanno N, Bianco C, De luca A \& Salomon DS 2001 The role of EGF-related peptides in tumor growth. Frontiers in Bioscience 6 d685-d707.

Normanno N, De Luca A, Bianco C, Strizzi L, Mancino M, Maiello MR, Carotenuto A, De Feo G, Caponigro F \& Salomon DS 2006 Epidermal growth factor receptor (EGFR) signaling in cancer. Gene 366 2-16.

Orian JM, Tamakoshi K, Mackay IR \& Brandon MR 1990 New murine model for hepatocellular carcinoma: transgenic mice expressing metallothionein-ovine growth hormone fusion gene. Journal of the National Cancer Institute 82 393-398.

Pollak M, Blouin MJ, Zhang JC \& Kopchick JJ 2001 Reduced mammary gland carcinogenesis in transgenic mice expressing a growth hormone antagonist. British Journal of Cancer $\mathbf{8 5}$ 428-430.

Quesnelle KM, Boehm AL \& Grandis JR 2007 STAT-mediated EGFR signaling in cancer. Journal of Cellular Biochemistry 102 311-319 (Review).

Raccurt M, Lobie PE, Moudilou E, Garcia-Caballero T, Frappart L, Morel G \& Mertani HC 2002 High stromal and epithelial human gh gene expression is associated with proliferative disorders of the mammary gland. Journal of Endocrinology 175 307-318.

Santos A, Yusta B, Fernandez-Moreno MD \& Blazquez E 1994 Expression of insulin-like growth factor-I (IGF-I) receptor gene in rat brain and liver during development and in regenerating adult rat liver. Molecular and Cellular Endocrinology 101 85-93.

Siegel G \& Tomer Y 2005 Is there an association between acromegaly and thyroid carcinoma? A critical review of the literature Endocrine Research 31 $51-58$.

Snibson KJ 2002 Hepatocellular kinetics and the expression of growth hormone $(\mathrm{GH})$ in the livers and liver tumours of $\mathrm{GH}$-transgenic mice. Tissue \& Cell 34 88-97.

Stofega MR, Herrington J, Billestrup N \& Carter Su C 2000 Mutation of the SHP-2 binding site in growth hormone $(\mathrm{GH})$ receptor prolongs GH-promoted tyrosil phophorylation of GH receptor, JAK2 and STAT5B. Molecular Endocrinology 14 1338-1350.

Styles JA, Kelly MD, Pritchard NR \& Foster JR 1990 Effects produced by the non-genotoxic hepatocarcinogen methylclofenapate in dwarf mice: peroxisome induction uncoupled from DNA synthesis and nuclearity changes. Carcinogenesis 3 387-391.

Swanson SM \& Unterman TG 2002 The growth hormone-deficient Spontaneous Dwarf rat is resistant to chemically induced mammary carcinogenesis. Carcinogenesis 23 977-982.

Wanke R, Hermanns W, Folger S, Wolf E \& Brem G 1991 Accelerated growth and visceral lesions in transgenic mice expressing foreign genes of the growth hormone family: an overview. Pediatric Nephrology 5 513-521.

Waters MJ, Hoang HN, Fairlie DP, Pelekanos RA \& Brown RJ 2006 New insights into growth hormone action. Journal of Molecular Endocrinology 36 1-7. 
Webb SM, Casanueva F \& Wass JA 2002 Oncological complications of excess GH in acromegaly. Pituitary 5 21-25.

Woelfle J \& Rotwein P 2004 In vivo regulation of growth hormone-stimulated gene transcription by STAT5b. American Journal of Physiology. Endocrinology and Metabolism 286 E393-E401.

Wu SL, Kim J, Bandle RW, Liotta L, Petricoin E \& Karger BL 2006 Dynamic profiling of the post-translational modifications and interaction partners of epidermal growth factor receptor signaling after stimulation by epidermal growth factor using Extended Range Proteomic Analysis (ERPA).

Molecular and Cellular Proteomics 9 1610-1627.

Yamashita H, Iwase H, Toyama T \& Fujii Y 2003 Naturally occurring dominant-negative Stat5 suppresses transcriptional activity of estrogen receptors and induces apoptosis in T47D breast cancer cells. Oncogene $\mathbf{2 2}$ 1638-1652.

Yamauchi T, Ueki K, Tobe K, Tamemoto H, Sekine N, Wada M, Honjo M, Takahashi M, Takahashi T, Hirai H et al. 1997 Tyrosine phosphorylation of the EGF receptor by the kinase Jak2 is induced by growth hormone. Nature 390 91-96.

Yamauchi T, Ueki K, Tobe K, Tamemoto H, Sekine N, Wada M, Honjo M, Takahashi M, Takahashi T, Hirai H et al. 1998 Growth hormone-induced tyrosine phosphorylation of EGF receptor as an essential element leading to MAP kinase activation and gene expression. Endocrine Journal 45 S27-S31.
Yamauchi T, Yamauchi N, Ueki K, Sugiyama T, Waki H, Miki H, Tobe K, Matsuda S, Tsushima T, Yamamoto T et al. 2000 Constitutive tyrosine phosphorylation of ErbB-2 via Jak2 by autocrine secretion of prolactin in human breast cancer. Journal of Biological Chemistry 275 33937-33944.

Yu H \& Jove R 2004 The STATs of cancer - new molecular targets come of age. Nature Reviews. Cancer 4 97-105.

Zhang T, Ma J \& Cao X 2003 Grb2 regulates Stat3 activation negatively in epidermal growth factor signalling. Biochemical Journal 376 457-464.

Zhou Y, Xu BC, Maheshwari HG, He L, Reed M, Lozykowski M, Okada S, Cataldo L, Coschigamo K, Wagner TE et al. 1997 A mammalian model for Laron syndrome produced by targeted disruption of the mouse growth hormone receptor/binding protein gene (the Laron mouse). PNAS 94 13215-13220.

Zhu T, Goh EL, Graichen R, Ling L \& Lobie PE 2001 Signal transduction via the growth hormone receptor. Cellular Signalling 13 599-616.

Received in final form 11 December 2009

Accepted 23 December 2009

Made available online as an Accepted Preprint 23 December 2009 